\title{
Sedation under JCI standard
}

\author{
Tae Dong Kweon \\ Department of Anesthesiology and Pain Medicine, Severance Hospital, Yonsei University College of Medicine
}

The practice of anesthesia and sedation continues to expand beyond the operating room and now includes the gastroenterology suite, magnetic resonance imaging suites, and the cardiac catheterization laboratory. Nonanesthesiologists frequently administer sedation, in part because of a lack of available anesthesiologists and economic aspect, which emphasizes the safety of sedation. The Joint Commission International (JCI) set a standard responding to this issue indicating that qualified individuals who have drug and monitoring knowledge as well as airway management skills can only administer sedating agents. In Korea, the Ministry of Health and Welfare developed new sedation standards for hospital evaluation, which is similar to the JCI standards. This review intends to help with the understanding of the JCI sedation standard and compare it to the Korean sedation standard. (Korean J Anesthesiol 2011; 61: 190-194)

Key Word: Sedation.

\section{Introduction}

Sedation represents a continuum of states ranging from minimal sedation (anxiolysis) to general anesthesia and may result in respiratory depression, loss of the patient's protective reflexes, and cardiopulmonary impairment.

The need for sedation has markedly increased with the development of new invasive diagnostic and minor surgical procedures outside of the operating room setting, which makes it important to provide patients with the benefits of sedation while minimizing adverse responses.

Safe sedation requires a systemic presedation evaluation, particularly for the airway, and a clear understanding of the medications used and skills in airway management, which the Joint Commission International (JCI) standards pursue.

Severance hospital has accepted the JCI standards and has revised all sedation policies to the JCI standards.

The JCI standard states that the chief of the anesthesiology department is in charge of instituting the sedation practice policy and protocol.

A sedation committee must be established, which should consist of physicians from various departments in which sedation is performed as committee members and the chief of the anesthesiology department as the committee president.

This committee has the responsibility to determine the requirements for qualified individuals to sedate, obtain

Received: March 29, 2011. Revised: April 20, 2011. Accepted: April 27, 2011.

Corresponding author: Tae Dong Kweon, M.D., Department of Anesthesiology and Pain Medicine, Severance Hospital, Yonsei University College of Medicine, 134, Shinchon-dong, Seodaemun-gu, Seoul 120-752, Korea. Tel: 82-2-2228-2428, Fax: 82-2-2227-7897, E-mail: ysanesthe71@hotmail.com

(c) This is an open-access article distributed under the terms of the Creative Commons Attribution Non-Commercial License (http:// creativecommons.org/licenses/by-nc/3.0/), which permits unrestricted non-commercial use, distribution, and reproduction in any medium, provided the original work is properly cited. 
Table 1. JCI Standard of Sedation

Sedation Care Standard

ASC.3 Policies and procedures guide the care of patients undergoing moderate and deep sedation

Measurable Element of ASC.3

1. Appropriate policies and procedures, addressing at least elements a) through $\mathrm{f}$ ) found in the intent statement, guides the care of patients undergoing moderate and deep sedation.

2. The qualified individual identified in ASC2 participates in the development of the policies and procedures.

3. There is a presedation assessment based on organizational policy to evaluate risk and appropriateness of the sedation for patients.

4. The qualified practitioner responsible for the sedation is qualified in at least elements $\mathrm{g}$ ) through $\mathrm{k}$ ) in the intent statement

g) techniques of various modes of sedation, h) appropriate monitoring

i) response to complications $\quad$ j) use of reversal agents. and

k) at least basic life support

5. A qualified individual monitors the patient during sedation and during the recovery period from sedation and documents the monitoring. 6. Moderate and deep sedation are administrated according to hospital policy.

informed consent, conduct a presedation assessment, and procure the proper equipment and monitoring (Table 1).

\section{Sedation Policy}

\section{Scope of sedation}

Sedation in the JCI standards is classified into four categories, including minimal sedation, moderate sedation, deep sedation, and anesthesia.

Sedation policy usually does not apply to minimal sedation and anesthesia or for sedation used for mechanically ventilated patients, psychological management, and sometimes for direct management of the anesthesiologist, which can change according to the policies of different institutes.

\section{Requirements of a qualified individual to practice sedation}

The JCI standards require that a qualified individual for sedation should have the ability to perform the various modes of sedation, monitor the patients, and manage the adverse responses, particularly those relating to the airway, as well as the medications, including reversal agents and basic life support (BLS).

The members of the education subcommittee should consider developing a physician and nurse education program.

The education program will consist of a lecture on sedation techniques, medications, responses to complications, and an appropriate monitoring and practice program for airway management techniques.

The institute will provide a VCR systemrather than a lecture for convenient access at anytime. Other institutes have held a sedation special week festival to increase the importance of sedation during the education process.

It is recommended that lectures will be provided on a departmental basis, because the necessary knowledge for sedation is slightly different in each department.

Practicing airway management technique will be implemented by the education subcommittee or replaced by advanced cardiac life support (ACLS) or pediatric advanced life support (PALS), if these programs have been running regularly.

Although JCI has set the standards for sedation practice as at least BLS, they require advanced airway management techniques.

There is no rule in the JCI standards as to whether anesthesiologists should be qualified for sedation without participation in an education program.

The sedation qualification requirement for anesthesiologists will be defined by the sedation committee.

There is no definite standard for renewing the sedation qualification in the JCI standards.

At Severance hospital, physicians should renew their sedation qualification every 2 years, because practice programs such as BLS, ACLS, and PALS have 2-year validation period.

If your institute has its own practice program, you can determine the required time for renewing the qualification.

The sedation policy should be defined in terms of safety, so a qualified physician should consider consulting with an anesthesiologist prior to sedation in the following situations; patient does not fulfill NPO criteria, or has severe cardiopulmonary, neurological, or other organ system disease, which may present a significant hazard and lead to a potentially difficult airway.

\section{Sedation location, equipment and monitoring}

A pre-sedation assessment after obtaining informed consent should be performed in a private room prior to beginning sedation.

The sedation location and recovery room should be equipped with appropriate monitoring and emergency resuscitation systems.

Routine monitoring should include heart rate, EKG, and 
pulse oximetry.

End tidal $\mathrm{CO}_{2}$ monitoring may be routine monitoring or not based on the sedation policy at your institute.

Emergency equipment must be immediately available at every location where moderate or deep sedation is administered or recovery occurs and include at least the following: defibrillator, suction device, oxygen, airway management tools, emergency drugs, intubation equipment, and an EKG monitor.

\section{Documentation}

Informed consent, pre-sedation assessment, monitoring during the procedure, and recovery records should be documented.

Informed consent for sedation includes the sedation method, necessities, alternatives, success rates, complications, and cautions.

A presedation evaluation and assessment will be performed by a physician and nurse and include a medical history and current medications, prior history of adverse reactions to sedation or anesthesia, airway status and NPO time, and vital signs.

If the patient has a difficult airway, it is helpful to consult with the anesthesiology department.

A premedication and sedation plan should be completed before proceeding with sedation.

The JCI standards define documentation of the monitored parameters, but do not mention specific parameters. Although the American Society of Anesthesiology (ASA) guidelines recommend that vital signs, respiratory rate, and patient response to verbal commands should be routinely monitored and recorded, your institute can select the routine monitoring parameters for procedure and recovery.
End tidal $\mathrm{CO}_{2}$ monitoring may be beneficial for deep sedation and for infants with a difficult airway, particularly when a procedure room, such as the magnetic resonance imaging (MRI) room is remotely located.

Monitoring and documentation will continue every $10 \mathrm{~min}$ during the procedure and for $30 \mathrm{~min}$ after entering the recovery room.

Patients receiving reversal agents will be monitored for a minimum of 2 hours.

Routine use of reversal agentsis discouraged.

The patient should be discharged from the recovery area by a qualified individual using established criteria.

The Aldrete score has been used at Severance hospital as the discharge criteria.

If discharged, the patient must be provided with written instructions regarding diet, medications, activities, and signs or symptoms of complications when they occur.

If the patient is transferred to the intensive care unit, they should receive an equal level of patient care (Table 2).

\section{Sedation guidelines}

Sedation is applied to both children and adults in many fields, such as endoscopy, MRI, and bronchoscopy.

Sedation of an infant differs from sedating an adult in terms of the drugs used, NPO time, and discharge criteria.

This difference should be stated in the sedation policy, so a separate policy will be developed for the pediatric and adult sedation populations.

The definition of a pediatric patient is a critical issue that may provoke conflict among medical departments.

Another difference is that the requirement of the qualified physician for sedation is different between pediatric and adult

Table 2. Aldrete Scoring Guide

\begin{tabular}{|c|c|c|}
\hline Aldrete scoring guide & & On discharge \\
\hline Able to move four extremities voluntarily or on command = 2 & \multirow[t]{3}{*}{ Activity } & \\
\hline Able to move two extremities voluntarily or on command = 1 & & \\
\hline Able to move $0-1$ extremity voluntarily or on command $=0$ & & \\
\hline Deep Breathing and Cough $=2$ & \multirow[t]{3}{*}{ Respiration } & \\
\hline Dyspnea or limited breathing $=1$ & & \\
\hline Apneic $=0$ & & \\
\hline $\mathrm{BP}=20 \mathrm{~mm}$ of preanesthetic level $=2$ & \multirow[t]{3}{*}{ Circulation } & \\
\hline $\mathrm{BP}=20-50 \mathrm{~mm}$ of preanesthetic level $=1$ & & \\
\hline $\mathrm{BP}=50 \mathrm{~mm}$ of preanesthetic level $=0$ & & \\
\hline Fully awake $=2$ & \multirow[t]{3}{*}{ Consciousness } & \\
\hline Arousable on calling $=1$ & & \\
\hline Not responding $=0$ & & \\
\hline Pink or normal skin color $=2$ & \multirow[t]{4}{*}{ Color } & \\
\hline Pale, dusky, blotchy, jaundiced, other $=1$ & & \\
\hline Cyanotic $=0$ & & \\
\hline Total score & & \\
\hline
\end{tabular}


sedation.

Usually, the physician in charge of pediatric sedation should be skilled in PALS, whereas the physician in charge of adult sedation shouldbe skilled in adult advanced life support.

Childrenare often sedated to control behavior to allow for safe completion of a procedure. A child's ability to control his or her own behavior and to cooperate during a procedure depends on their chronological and developmental age. Children $<6$ years or those with a developmental delay often require a deeper level of sedation to control their behavior.

Indications for pediatric sedation include endoscopy, liver and kidney biopsy, an electrophysiological study, angiography, bone marrow study, and MRI.

Midazolam and propofol are mainly used for loss of consciousness, whereas fentanyl, ketamine, remifentanil, or pethidine are given additionally for painful procedures.

Midazolam combined with ketamine is the safest regimen for infant sedation [1].

This combination can reduce the safety range, so a reduced dosage should be used [2].

Endoscopy for infants has been conducted frequently with a combination of midazolam and fentanyl $[3,4]$.

Propofol can be a good alternative when considering recovery time and sedation quality [5].

Ketamine increases the incidence of laryngospasm during an upper endoscopy $[6,7]$ but can be an alternative to lower GI endoscopy.

Chloral hydrate, ketamine, midazolam, propofol, and dexmedetomidine are useful for sedation during non painful procedures such as computed tomography and MRI.

Although chloral hydrate is safe, it is not recommended, because the failure rate is up to $20 \%$, which is economically costly $[8,9]$.

Midazolam is not suitable for MRI imaging because the sedation duration is approximately $30 \mathrm{~min}$ or longer [10].

Ketamine is not suitable for MRI sedation because hypertonicity, hypertension, and re-emergence phenomenon occur more frequently. But, in patients with difficult airways or in cardiac patients, ketamine has the advantage of airway patency and hemodynamic stability [11].

Propofol has a rapid onset and short duration, which is effective for edating children undergoing an MRI procedure. However, caution should be taken because $<1 \%$ respiratory compromise has been reported.

Dexmedetomidine is a selective alpha-2 agonist, which is recommended by the ASA as a sedative that can be used by non-anesthesiologists [12].

This drug features no relevant respiratory effects, low blood pressure, or bradycardia.

Some investigators have found no differences in successful
MRI scanning between patients sedated with dexmedetomidine or propofol $[13,14]$.

Dexmedetomidine can be injected intramuscularly, which is an advantage when administering a sedative to an infant with no intravenous line.

As access to the patient is limited in an MRI setting and respiratory movement can not be seen directly, the physician should consider the risks and must be very experienced in airway management, particularly when testing disabled children or newborns.

It is recommended that supplemental oxygen be used and end tidal $\mathrm{CO}_{2}$ be monitored to increase safety.

Adult sedation for MRIs, patients with claustrophobia or a movement disorder, as well as the cardiac catheterization lab, endoscopy, and bronchoscopy.

Midazolam is used frequently for adult endoscopy throughout the world, because it has a rapid onset of action within $2 \mathrm{~min}$, but the elimination half life is between 1 and 2.8 hours. Propofol cause hypnosis within 40 seconds and has an antiemetic effect with a shorter duration of action.

A meta-analysis demonstrated that propofol provides significant advantages over benzodiazepines to induce and maintain adequate sedation during endoscopic procedures $[15,16]$. When a recovery room is not large enough for a sedation procedure, propofol is preferred because the effect of propofol wears off more rapidly than that of midazolam.

\section{Conclusion}

Sedation under the JCI standards is mainly based on the ASA recommendations.

However, because the mission of the JCI standard is to improve the quality and safety of care in the international community, JCI defines the minimum requirements.

Following this standard will provide your hospital with the most efficient, safe, and economically efficient policy.

\section{References}

1. Neuhäuser C, Wagner B, Heckmann M, Weigand MA, Zimmer KP. Analgesia and sedation for painful interventions in children and adolescents. Dtsch Arztebl Int 2010; 107: 241-7.

2. Elitsur Y, Blankenship P, Lawrence Z. Propofol sedation for endoscopic procedures in children. Endoscopy 2000; 32: 788-91.

3. Mamula P, Markowitz JE, Neiswender K, Zimmerman A, Wood S, Garofolo M, et al. Safety of intravenous midazolam and fentanyl for pediatric GI endoscopy prospective study of 1578 endoscopies. Gastrointest Endosc 2007; 65: 203-10.

4. Fredette ME, Lightdale JR. Endoscopic sedation in pediatric practice. Gastrointest Endosc Clin N Am 2008; 18: 739-51.

5. Lightdale JR, Valim C, Newburg AR, Mahoney LB, Zgleszewski 
S, Fox VL. Efficiency of propofol versus midazolam and fentanyl sedation at a pediatric teaching hospital: a prospective study. Gastrointest Endosc 2008; 67: 1067-75.

6. Aggarwal A, Ganguly S, Anand VK, Patwari AK. Efficacy and safety of intravenous ketamine for sedation and analgesia during pediatric endoscopic procedures. Indian Pediatr 1998; 35: 1211-14.

7. Gilger MA, Spearman RS, Dietrich CL, Spearman G, Wilsey MJ Jr, Zayat MN. Safety and effectiveness of ketamine as a sedative agent for pediatric GI endoscopy. Gastrointest Endosc 2004; 59: 659-63.

8. Cortellazzi P, Lamperti M, Minati L, Falcone C, Pantaleoni C, Caliroli D. Sedation of neurologically impaired children undergoing MRI: a sequential approach. Paediatr Anaesth 2007; 17: 630-6.

9. Low E, O'Driscoll M, MacEneaney P, O'Mahony O. Sedation with oral chloral hydrate in children undergoing MRI scanning. Ir Med J 2008; 101: 80-2.

10. Schulte-Uentrop L, Goepfert MS. Anaethesia or sedation for MRI in children. Curr Opin Anaesthesiol 2010; 23: 513-7.

11. Vardy JM, Dignon N, Mukherjee N, Sami DM, Balachandran G, Taylor S. Audit of the safety and effectiveness of ketamine for procedural sedation in the emergency department. Emerg Med J 2008; 25: 579-82.

12. American Society of Anesthesiologists Task Force on sedation and analgesia. Practice guideline for sedation and analgesia by NonAnesthesiologists. Anesthesiology 2002; 96: 1004-17.

13. Mason KP, Zurakowski D, Zgleszewski SE, Robson CD, Carrier M, Hickey PR, et al. High dose dexmedetomidine as the sole sedative for pediatric MRI. Paediatr Anaesth 2008; 18: 403-11.

14. Mahmoud M, Gunter J, Donnelly LF, Wang Y, Nick TG, Sadhasivam S. A comparison of dexmedetomidine with propofol for magnetic resonance imaging sleep studies in children. Anesth Analg 2009; 109: 745-53.

15. Qadeer MA, Vargo JJ, Khandwala F, Lopez R, Zuccaro G. Propofol versus traditional sedative agents for gastrointestinal endoscopy; a meta analysis. Clin Gastroenterol Hepatol 2005; 3: 1049-56.

16. Cohen LB, Hightower CD, Wood DA, Miller KM, Aisenberg J. Moderate level sedation during endoscopy: a prospective study using low-dose propofol, meperidie/fentanyl, and midazolam. Gastrointest Endosc 2004; 59: 795-803. 